\title{
Auto-CaseRec: Automatically Selecting and Optimizing Recommendation-Systems Algorithms
}

\author{
Srijan Gupta, Joeran Beel \\ Trinity College Dublin, Ireland \& The University of Siegen, Germany \\ guptasr@tcd.ie, joeran.beel@uni-siegen.de
}

\begin{abstract}
The advances in the field of Automated Machine Learning (AutoML) have greatly reduced human effort in selecting and optimizing machine learning algorithms. These advances, however, have not yet widely made it to Recommender-Systems libraries. We introduce Auto-CaseRec, a Python framework based on the CaseRec recommendersystem library ${ }^{1}$. Auto-CaseRec provides automated algorithm selection and parameter tuning for recommendation algorithms. An initial evaluation of Auto-CaseRec against the baselines shows an average $13.88 \%$ improvement in RMSE for the Movielens100K dataset and an average $17.95 \%$ improvement in RMSE for the Last.fm dataset.
\end{abstract}

\section{Introduction}

There exist numerous machine learning algorithms as well as data processing techniques. Different machine learning algorithms are tunable according to different sets of hyerparameters. The choice of algorithms and parameters depends on a plethora of design decisions. For a domain scientist who does not have extensive knowledge of machine learning, navigating through these choices is a much difficult task. The AutoML community has been successful in developing frameworks like Auto-sklearn [Feurer et al., 2015] and AutoWEKA [Thornton et al., 2013; Kotthoff et al., 2017] that make and apply these decisions automatically, efficiently and optimally for a given scenario, while the user is just required to provide the data. AutoML approaches use advanced Hyper - Parameter Optimization (HPO) techniques to find an optimal pipeline. An end-to-end pipeline consists of data processing, algorithm selection, and algorithm tuning. While Auto-WEKA and Auto-sklearn utilize the model-based approached SMAC [Hutter et al., 2011], ML-Plan [Mohr et al., 2018] uses Hierarchical Task Planning, an AI-planning technique, and TPOT [Olson and Moore, 2019] uses evolutionary algorithms.

The need for such systems is there in the recommender systems domain as well. It has been shown that the reproducibility of research on recommender systems is rarely

\footnotetext{
${ }^{1}$ https://github.com/caserec/CaseRecommender
}

given, where even small changes in scenarios can lead to wide discrepancies in results [Beel et al., 2016]. AutoML frameworks have been said to provide three-fold benefits [Hutter et al., 2019]: they reduce human effort, improve system performance and improve reproducibility in scientific research. Even so, no such frameworks exist for the recommender systems domain. Recommender-systems libraries typically do not offer such functionality including Surprise [Hug, 2017], CaseRec [da Costa and Manzato, 2016], OpenRec [Yang et al., 2018], Spotlight [Kula, 2017], NeuRec [Zhang et al., 2018], MyMediaLite [Gantner et al., 2011], LensKit [Ekstrand et al., 2011], LibRec [Guo et al., 2015]

We introduce Auto-CaseRec ${ }^{2}$, a Python framework that provides automatic algorithm selection and hyperparameter tuning for a variety of recommendation algorithms. Auto-CaseRec saves developers from the arduous process of finding the right algorithm configuration for their problem. The framework also enables greater research reproducibility by serving as a robust tool for evaluating baseline algorithms. Auto-CaseRec extends the Case Recommender library [da Costa and Manzato, 2016], a flexible Python library that provides 10 item-recommendation and 15 ratingprediction algorithms for recommender system datasets. For finding a set of algorithm and parameters, Auto-CaseRec provides 3 optimization strategies: Tree of Parzen Estimators (TPE) [Bergstra et al., 2013a], Adaptive-TPE and Random Search, implemented from the Hyperopt [Bergstra et al., 2013b] Python library for Hyper-Parameter Optimization. Auto-CaseRec is - after LibRec-Auto [Mansoury et al., 2018; Mansoury and Burke, 2019] and along with Auto-Surprise [Anand and Beel, 2020] - among the first recommendation system libraries that applies advanced AutoML techniques to select and optimize recommendation algorithms.

\section{Design}

The workflow of Auto-CaseRec is shown in Figure 1. The user creates an AutoEstimator class object with the appropriate input parameters. The search algorithm is initiated using the fit() method of the AutoEstimator object. The search algorithm of Auto-CaseRec operates as follows: A search space consisting of various algorithms and their parameters is continuously sampled according to the optimization strategy. In

\footnotetext{
${ }^{2}$ https://github.com/BeelGroup/Auto-CaseRec
} 


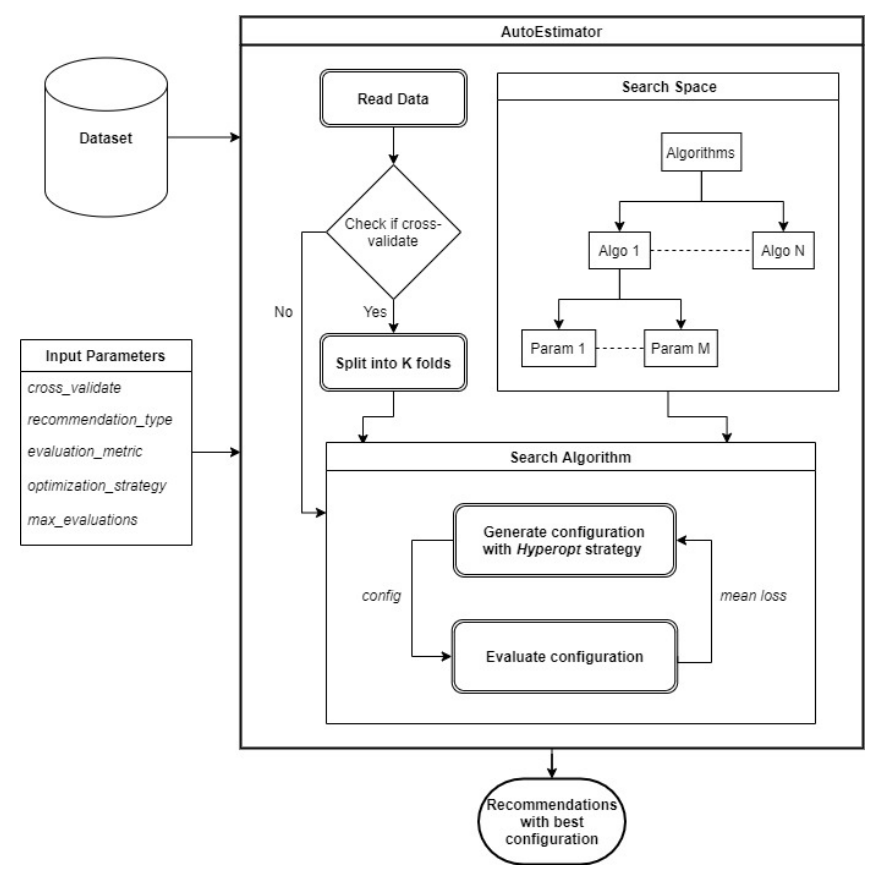

Figure 1: Workflow of Auto-CaseRec

each iteration, the sampled algorithm is fit on the dataset with the sampled parameters. Then, K-Fold Cross Validation is performed and the mean error rate is returned to the optimization strategy. The search algorithm terminates after a specified number of evaluations and returns the best algorithm and its parameters for the scenario.

The categories of input parameters that the user can provide to the AutoEstimator class object are:

- Data processing: The user is required to specify the data-path, whether to perform K-fold cross-validation and the number of folds $\mathrm{K}$.

- Recommendation Algorithm: The user chooses the type of recommendation algorithm and evaluation metric. Auto-CaseRec implements two types of recommendation algorithms from the Case Recommender library.

- Rating prediction algorithms use explicit feedback to estimate user ratings for unrated objects. They are evaluated using metrics like RMSE and MAE.

- Item recommendation algorithms use implicit feedback, i.e., no explicit knowledge of user preferences, to produce recommendations. They are evaluated using Precision, Recall, etc.

- Optimization strategy: Using these parameters, the user can set the maximum number of configuration evaluations, the optimization strategy as well as a custom search space if he wants to limit the search.

The framework also implements multiple optimization strategies from the Hyperopt library, including, TPE, AdaptiveTPE and Random Search. The users can choose a single optimization strategy to use in the search algorithm or multiple strategies together by specifying a split.

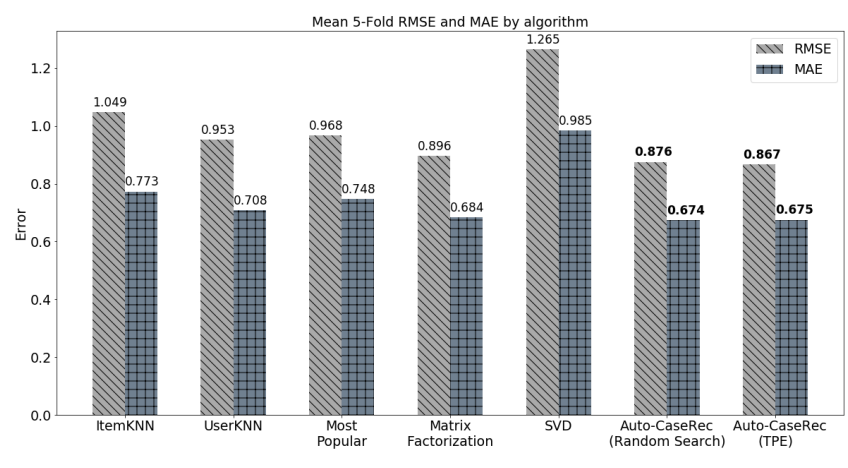

Figure 2: MovieLens100K Evaluation Results

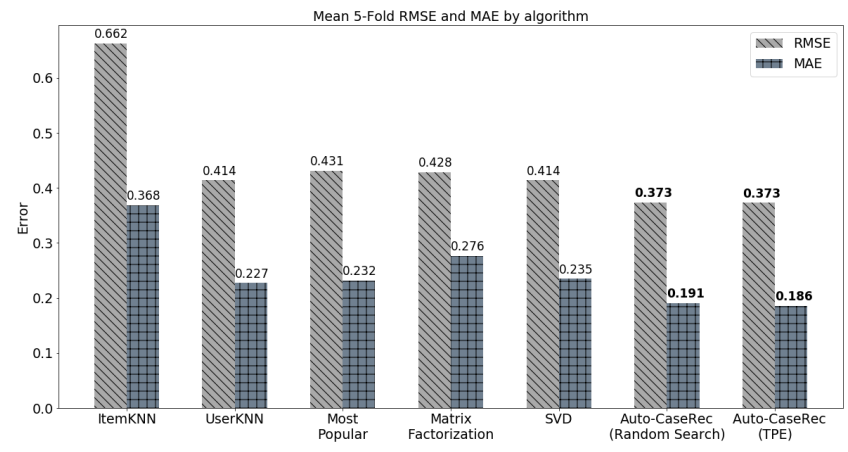

Figure 3: Last.fm Evaluation Results

\section{Evaluation}

We compared Auto-CaseRec with default implementations of 5 rating prediction algorithms from Case Recommender. The tests were conducted on the Movielens $100 \mathrm{~K}$ dataset and the Last.fm music rating dataset. Auto-CaseRec was run with both TPE and Random Search, each for a maximum of 100 iterations. The evaluation metrics used were the Root Mean Squared Error (RMSE) and Mean Absolute Error (MAE), averaged across 5 folds. The best algorithms and parameters produced by Auto - CaseRec outperformed the baseline algorithms with an average improvement of $13.88 \%$ in RMSE and $12.02 \%$ in MAE for the Movielens $100 \mathrm{~K}$ dataset (Figure 2), and average improvement of $17.95 \%$ in RMSE and $27.3 \%$ in MAE for the Last.fm dataset (Figure 3).

\section{Conclusion and Outlook}

We introduced Auto-CaseRec that provides automated algorithm selection and hyperparameter tuning for recommender system algorithms. The evaluation shows that Auto-CaseRec delivers notable improvements in both RMSE and MAE over the simple baselines. However, in the future more evaluation is needed, especially against stronger baselines. Only then we will be able to say with certainty if and to what extent Auto-CaseRec is better (faster and/or higher performance) than "normal" recommender-system libraries. 


\section{References}

[Anand and Beel, 2020] Rohan Anand and Joeran Beel. Auto-surprise: An automated recommender-system (autorecsys) library with tree of parzens estimator (tpe) optimization. In 14th ACM Conference on Recommender Systems (RecSys), 2020.

[Beel et al., 2016] Joeran Beel, Corinna Breitinger, Stefan Langer, Andreas Lommatzsch, and Bela Gipp. Towards reproducibility in recommender-systems research. User modeling and user-adapted interaction, 26(1):69-101, 2016.

[Bergstra et al., 2013a] James Bergstra, Dan Yamins, and David D Cox. Hyperopt: A python library for optimizing the hyperparameters of machine learning algorithms. In Proceedings of the 12th Python in science conference, pages 13-20. Citeseer, 2013.

[Bergstra et al., 2013b] James Bergstra, Daniel Yamins, and David Daniel Cox. Making a science of model search: Hyperparameter optimization in hundreds of dimensions for vision architectures. 2013.

[da Costa and Manzato, 2016] Arthur F da Costa and Marcelo G Manzato. Case recommender: A recommender framework. In Anais Estendidos do XXII Simpósio Brasileiro de Sistemas Multimídia e Web, pages 99-102. SBC, 2016.

[Ekstrand et al., 2011] Michael D Ekstrand, Michael Ludwig, Jack Kolb, and John T Riedl. Lenskit: a modular recommender framework. In Proceedings of the fifth ACM conference on Recommender systems, pages 349350. ACM, 2011.

[Feurer et al., 2015] Matthias Feurer, Aaron Klein, Katharina Eggensperger, Jost Springenberg, Manuel Blum, and Frank Hutter. Efficient and robust automated machine learning. In Advances in neural information processing systems, pages 2962-2970, 2015.

[Gantner et al., 2011] Zeno Gantner, Steffen Rendle, Christoph Freudenthaler, and Lars Schmidt-Thieme. Mymedialite: A free recommender system library. In Proceedings of the fifth ACM conference on Recommender systems, pages 305-308. ACM, 2011.

[Guo et al., 2015] Guibing Guo, Jie Zhang, Zhu Sun, and Neil Yorke-Smith. Librec: A java library for recommender systems. In Posters, Demos, Late-breaking Results and Workshop Proceedings of the 23rd International Conference on User Modeling, Adaptation and Personalization, 2015.

[Hug, 2017] Nicolas Hug. Surprise, a Python library for recommender systems. http://surpriselib.com, 2017.

[Hutter et al., 2011] Frank Hutter, Holger H Hoos, and Kevin Leyton-Brown. Sequential model-based optimization for general algorithm configuration. In International conference on learning and intelligent optimization, pages 507-523. Springer, 2011.
[Hutter et al., 2019] Frank Hutter, Lars Kotthoff, and Joaquin Vanschoren. Automated Machine Learning. Springer, 2019.

[Kotthoff et al., 2017] Lars Kotthoff, Chris Thornton, Holger H Hoos, Frank Hutter, and Kevin Leyton-Brown. Auto-weka 2.0: Automatic model selection and hyperparameter optimization in weka. The Journal of Machine Learning Research, 18(1):826-830, 2017.

[Kula, 2017] Maciej Kula. Spotlight. https://github.com/ maciejkula/spotlight, 2017.

[Mansoury and Burke, 2019] Masoud Mansoury and Robin Burke. Algorithm selection with librec-auto. In Joeran Beel and Lars Kotthoff, editors, Proceedings of The 1st Interdisciplinary Workshop on Algorithm Selection and Meta-Learning in Information Retrieval (AMIR). CEURWS, 2019.

[Mansoury et al., 2018] Masoud Mansoury, Robin Burke, Aldo Ordonez-Gauger, and Xavier Sepulveda. Automating recommender systems experimentation with librecauto. In Proceedings of the 12th ACM Conference on Recommender Systems, pages 500-501. ACM, 2018.

[Mohr et al., 2018] Felix Mohr, Marcel Wever, and Eyke Hüllermeier. Ml-plan: Automated machine learning via hierarchical planning. Machine Learning, 107(810):1495-1515, 2018.

[Olson and Moore, 2019] Randal S Olson and Jason H Moore. Tpot: A tree-based pipeline optimization tool for automating machine learning. In Automated Machine Learning, pages 151-160. Springer, 2019.

[Thornton et al., 2013] Chris Thornton, Frank Hutter, Holger H Hoos, and Kevin Leyton-Brown. Auto-weka: Combined selection and hyperparameter optimization of classification algorithms. In Proceedings of the 19th ACM SIGKDD international conference on Knowledge discovery and data mining, pages 847-855, 2013.

[Yang et al., 2018] Longqi Yang, Eugene Bagdasaryan, Joshua Gruenstein, Cheng-Kang Hsieh, and Deborah Estrin. Openrec: A modular framework for extensible and adaptable recommendation algorithms. In Proceedings of the Eleventh ACM International Conference on Web Search and Data Mining, pages 664-672, 2018.

[Zhang et al., 2018] Shuai Zhang, Lina Yao, Aixin Sun, Sen Wang, Guodong Long, and Manqing Dong. Neurec: On nonlinear transformation for personalized ranking. arXiv preprint arXiv:1805.03002, 2018. 